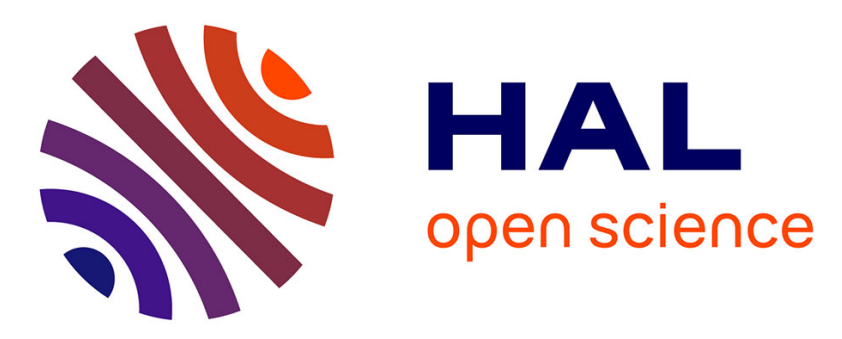

\title{
2D directional ramp filter
}

Feriel Khellaf, Nils Krah, Jean Michel Létang, Simon Rit

\section{To cite this version:}

Feriel Khellaf, Nils Krah, Jean Michel Létang, Simon Rit. 2D directional ramp filter. Physics in Medicine and Biology, 2020, 65 (8), pp.08NT01. 10.1088/1361-6560/ab7875 . hal-02486620

\section{HAL Id: hal-02486620 \\ https://hal.science/hal-02486620}

Submitted on 12 Oct 2020

HAL is a multi-disciplinary open access archive for the deposit and dissemination of scientific research documents, whether they are published or not. The documents may come from teaching and research institutions in France or abroad, or from public or private research centers.
L'archive ouverte pluridisciplinaire HAL, est destinée au dépôt et à la diffusion de documents scientifiques de niveau recherche, publiés ou non, émanant des établissements d'enseignement et de recherche français ou étrangers, des laboratoires publics ou privés. 


\title{
2D directional ramp filter
}

\author{
F. Khellaf ${ }^{1}$, N. Krah ${ }^{1,2}$, J. M. Létang ${ }^{1}$, S. Rit ${ }^{1}$ \\ ${ }^{1}$ University of Lyon, INSA-Lyon, Université Claude Bernard Lyon 1, UJM-Saint Etienne, CNRS, Inserm, CREATIS UMR \\ 5220, U1206, F-69008, LYON, France. \\ ${ }^{2}$ Institut de Physique des Deux Infinis de Lyon, UMR 5822, F-69622, Villeurbanne, France. \\ feriel.khellaf@creatis.insa-lyon.fr
}

\begin{abstract}
Usual tomographic reconstruction methods start by filtering projections before backprojecting the data. In some cases, inverting the filtering and the backprojection steps can be useful to preserve spatial information. In this paper, an intermediate between a filterbackproject and a backproject-filter approach is proposed, based on the extension of the usual ramp filter to two dimensions. To this end, an expression for a band-limited $2 \mathrm{D}$ version of the ramp filter is derived. We have tested this filter on simulated X-ray CT projections of a Shepp-Logan phantom and on proton CT list-mode data. We accurately reconstructed the X-ray CT and the proton CT data, although the reconstruction can be slightly noisier than a standard filtered backprojection in some cases. A slight improvement of the spatial resolution of proton CT images reconstructed with this $2 \mathrm{D}$ filter has been observed.
\end{abstract}

\section{INTRODUCTION}

The filtered backprojection (FBP) is the reference algorithm to reconstruct tomographic data. It consists in (1) filtering the projections with a 1D ramp filter and (2) backprojecting the result in the image domain. An alternative method, the backproject-filter or backproject-first $(\mathrm{BPF})$, starts with the backprojection before filtering in the 2D image domain. BPF approaches can be useful when dealing with certain modalities to avoid resampling. For example, in proton CT (pCT) (Figure 1 center), starting by backprojecting the protons along their non-linear paths could improve spatial resolution, compared with binning the list-mode data into projections (Zeng, 2007; Poludniowski et al., 2014, Rit et al., 2015). Similarly, time-of-flight information of positron emission tomography (PET) can be accounted for in the image domain (Watson, 2007) (Figure 1 right). Our objective is to develop such an approach by extending the usual 1D ramp filter in 2D. BPF methods usually rely on 2D radial filters (Zeng et al., 1994 Poludniowski et al., 2014). However, the backprojected image has an infinite support which is problematic during convolution since the backprojection matrix must have a finite size. The truncation will cause an offset in the reconstructed values. This effect can be minimized by using a very large backprojection matrix. Another possible backproject-first approach is based on the Hilbert transform inversion (Noo et al., 2004, Zeng, 2007; Rit et al., 2015), and involves a differentiation step.

In this paper, the idea is to (1) smear each projection in the image space, (2) filter the smeared projection with a $2 \mathrm{D}$ version of the $1 \mathrm{D}$ ramp filter and (3) integrate over all projection angles. A similar 2D version of the ramp filter has been proposed by Watson (2007) in the context of time-of-flight positron emission tomography (TOF PET), although the filter was sampled directly in the frequency domain causing sampling artifacts. A variation of this filter, also proposed by Watson (2007) and called CRG-CW (Convolved Ramp and Gaussian with Confidence Weighting), has been used for TOF direct reconstruction (Kao, 2008; Guo et al., 2011; Conti et al., 2013, Kao et al., 2010). Similarly to what is done for the 1D ramp filter, we sample the filter in the spatial domain to avoid sampling artifacts. We start by defining the 2D ramp filter in the case of parallel X-ray $\mathrm{CT}(\mathrm{xCT})$, derive its expression in the spatial domain, before applying this method to simulated $\mathrm{xCT}$ and $\mathrm{pCT}$ data. Although the main applications 


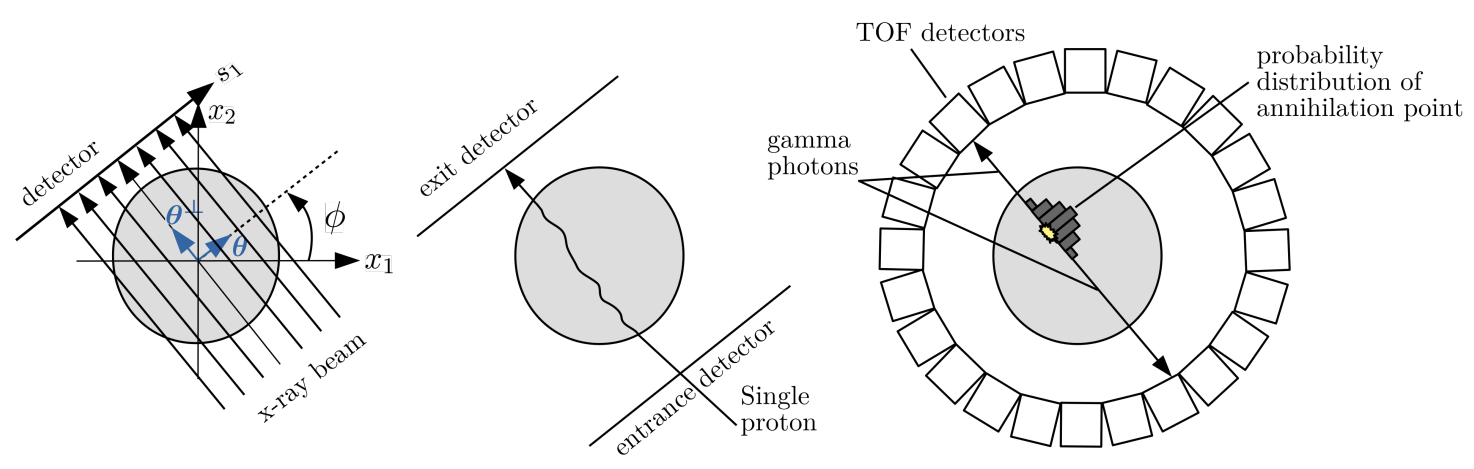

Figure 1: Parallel xCT geometry (left), pCT single tracking setup (middle), and TOF PET setup (right).

in mind for this method are pCT and TOF PET, the filter is mathematically demonstrated in a conventional $\mathrm{xCT}$ setting to avoid technicalities specific to proton/PET imaging. It is then applied to proton $\mathrm{CT}$ simulations.

\section{METHODS}

\subsection{Parallel FBP and 1D ramp filter}

An image $f\left(x_{1}, x_{2}\right)$, with $\left(x_{1}, x_{2}\right)$ the spatial coordinates, can be reconstructed from its parallel projections

$$
p_{\phi}\left(s_{1}\right)=\int_{\mathbb{R}} f\left(s_{1} \boldsymbol{\theta}+s_{2} \boldsymbol{\theta}^{\perp}\right) \mathrm{d} s_{2},
$$

where $s_{1}$ is the distance of the projection line from the center, $s_{2}$ is the position along the projection line, $\boldsymbol{\theta}=(\cos \phi, \sin \phi)$ and $\boldsymbol{\theta}^{\perp}=(-\sin \phi, \cos \phi)$ (see Figure 1). Using a filtered backprojection method, the image is reconstructed by

$$
f\left(x_{1}, x_{2}\right)=\int_{0}^{\pi} \tilde{p}_{\phi}\left(x_{1} \cos \phi+x_{2} \sin \phi\right) \mathrm{d} \phi
$$

where

$$
\tilde{p}_{\phi}\left(s_{1}\right)=\int_{\mathbb{R}} P_{\phi}\left(\sigma_{1}\right)\left|\sigma_{1}\right| e^{i 2 \pi \sigma_{1} s_{1}} \mathrm{~d} \sigma_{1},
$$

with $P_{\phi}\left(\sigma_{1}\right)$ defined as the Fourier transform (FT) of $p_{\phi}\left(s_{1}\right)$.

In practice, the filtered projections $\tilde{p}_{\phi}\left(s_{1}\right)$ are obtained using a band-limited version of the 1D ramp filter. A common choice proposed by Bracewell et al. (1967) and Ramachandran et al. (1971) is

$$
H\left(\sigma_{1}\right)= \begin{cases}\left|\sigma_{1}\right| & \text { if }-W<\sigma_{1}<W \\ 0 & \text { otherwise, }\end{cases}
$$

with $W$ the Nyquist frequency defined as $W=1 / 2 \tau$ with $\tau$ the sampling period of the projections $p_{\phi}$. Directly discretizing this expression to filter the projections in the frequency domain would cause an underestimation of the entire reconstructed values, because of the zero value attributed to the zero frequency bin (Kak et al., 1988, Crawford, 1991). In order to avoid this issue, the impulse response of the filter is calculated by taking its inverse Fourier transform

$$
h\left(s_{1}\right)=\int_{\mathbb{R}} H\left(\sigma_{1}\right) e^{i 2 \pi \sigma_{1} s_{1}} \mathrm{~d} \sigma_{1}=\frac{1}{2 \tau^{2}} \operatorname{sinc}\left(\frac{2 \pi s_{1}}{2 \tau}\right)-\frac{1}{4 \tau^{2}} \operatorname{sinc}^{2}\left(\frac{\pi s_{1}}{2 \tau}\right)
$$


and subsequently discretized by replacing $s_{1}=n \tau$, which yields

$$
h[n]= \begin{cases}\frac{1}{4 \tau^{2}}, & \text { if } n=0 \\ 0, & \text { if } n \text { even } \\ \frac{-1}{n^{2} \pi^{2} \tau^{2}}, & \text { if } n \text { odd }\end{cases}
$$

as was proposed by Ramachandran et al. (1971). The discrete Fourier transform (DFT) of this impulse response does not have a zero value at its zero frequency and allows an accurate reconstruction of the image values (Kak et al., 1988). Equation 6 is used to compute the 1D ramp filter used for FBP.

\subsection{From the 1D ramp filter to a $2 \mathrm{D}$ directional ramp filter}

From Equation 2, we see that the projections are filtered then smeared in the image space using the variable change $s_{1}=x_{1} \cos \phi+x_{2} \sin \phi$. We want to apply a $2 \mathrm{D}$ version of the ramp filter on the already smeared projections, i.e. do the variable change before filtering.

We recall that $\left(\sigma_{1}, \sigma_{2}\right)$ are the Fourier variables corresponding to $\left(s_{1}, s_{2}\right)$ in the spatial domain. We introduce the dirac $\delta$ function, using the property $\int_{\mathbb{R}} \delta\left(\sigma_{2}\right) e^{i 2 \pi \sigma_{2} s_{2}} \mathrm{~d} \sigma_{2}=1$, in Equation 3

$$
\tilde{p}_{\phi}\left(s_{1}\right)=\int_{\mathbb{R}} \int_{\mathbb{R}} \delta\left(\sigma_{2}\right) P_{\phi}\left(\sigma_{1}\right)\left|\sigma_{1}\right| e^{i 2 \pi\left(\sigma_{1} s_{1}+\sigma_{2} s_{2}\right)} \mathrm{d} \sigma_{1} \mathrm{~d} \sigma_{2}
$$

and, given the expression of the Fourier transform $P_{\phi}\left(\sigma_{1}\right)$ and the fact that formally $\delta\left(\sigma_{2}\right)=\int_{\mathbb{R}} e^{-i 2 \pi \sigma_{2} s_{2}} \mathrm{~d} s_{2}$, note that

$$
\delta\left(\sigma_{2}\right) P_{\phi}\left(\sigma_{1}\right)=\int_{\mathbb{R}} \int_{\mathbb{R}} p_{\phi}\left(s_{1}\right) e^{-i 2 \pi\left(\sigma_{1} s_{1}+\sigma_{2} s_{2}\right)} \mathrm{d} s_{1} \mathrm{~d} s_{2} .
$$

We use the following vector notations for $s_{1}=\boldsymbol{x} \cdot \boldsymbol{\theta}, s_{2}=\boldsymbol{x} \cdot \boldsymbol{\theta}^{\perp}$ and the corresponding Fourier variables $\sigma_{1}=\boldsymbol{\xi} \cdot \boldsymbol{\theta}$ and $\sigma_{2}=\boldsymbol{\xi} \cdot \boldsymbol{\theta}^{\perp}$, where $\boldsymbol{x}=\left(x_{1}, x_{2}\right)$ and $\boldsymbol{\xi}=\left(\xi_{1}, \xi_{2}\right)$. We also define $b_{\phi}: \mathbb{R}^{2} \rightarrow \mathbb{R}$, the backprojection of one projection, as

$$
b_{\phi}(\boldsymbol{x})=p_{\phi}(\boldsymbol{x} \cdot \boldsymbol{\theta})
$$

and $B_{\phi}(\boldsymbol{\xi})$ its Fourier transform. We call $b_{\phi}(\boldsymbol{x})$ a smeared projection. Using these definitions, we note that Equation 8 is the expression of the Fourier transform of $b_{\phi}(\boldsymbol{x})$, and Equation 7 becomes

$$
\tilde{b}_{\phi}(\boldsymbol{x})=\int_{\mathbb{R}^{2}} B_{\phi}(\boldsymbol{\xi})|\boldsymbol{\xi} \cdot \boldsymbol{\theta}| e^{i 2 \pi \boldsymbol{\xi} \cdot \boldsymbol{x}} \mathrm{d} \boldsymbol{\xi}
$$

and the image can then be reconstructed from

$$
f(\boldsymbol{x})=\int_{0}^{\pi} \tilde{b}_{\phi}(\boldsymbol{x}) \mathrm{d} \phi .
$$

This equation allows reconstructing the image in three steps

(1) Smear each projection in the image space, i.e. compute a $2 \mathrm{D}$ image $b_{\phi}(\boldsymbol{x})$ for each projection angle;

(2) Filter each 2D image $b_{\phi}(\boldsymbol{x})$ with the corresponding $2 \mathrm{D}$ filter $|\boldsymbol{\xi} \cdot \boldsymbol{\theta}|$ to obtain $\tilde{b}_{\phi}(\boldsymbol{x})$;

(3) Sum $\tilde{b}_{\phi}(\boldsymbol{x})$ over all angles $\phi$. 
Note that this 2D version of the ramp filter depends on $\phi$, hence the name directional ramp (DR) filter. By definition, the backprojection operation consists in smearing each projection in the image space, i.e. interpolating $b_{\phi}(\boldsymbol{x})$ from $p_{\phi}\left(s_{1}\right)$ using $b_{\phi}(\boldsymbol{x})=p_{\phi}(\boldsymbol{x} \cdot \boldsymbol{\theta})$ and summing over all projection angles, i.e. $\int_{0}^{\pi} b_{\phi}(\boldsymbol{x}) d \phi$. In the proposed method, we separate these two steps of the backprojection: steps (1) and (3) correspond to the backprojection and step (2) to the filtering. This method resembles a BPF approach since we start by smearing the projections in the image space, however the angular sum to compute the backprojected image is the last step as in an FBP. The usual 2D cone filter used in BPF methods is $\|\boldsymbol{\xi}\|$, and it is applied after the full backprojection since it is not direction dependent, unlike the DR filter which must be used before the angular sum as it depends on the projection angle. A similar filter to the 2D DR, called transverse ramp, has been used to reconstruct TOF PET data (Watson, 2007). It was directly sampled in the frequency domain, causing sampling artifacts due to the zero values along the line $\boldsymbol{\xi} \cdot \boldsymbol{\theta}=0$. We choose to calculate the filter's impulse response as in the $1 \mathrm{D}$ case to avoid such artifacts.

\subsection{Impulse response of the $2 \mathrm{D}$ directional ramp filter}

A band-limited version of the 2D DR filter is given by

$$
H_{\phi}(\boldsymbol{\xi})= \begin{cases}|\boldsymbol{\xi} \cdot \boldsymbol{\theta}| & \text { if }\left|\xi_{1}\right|,\left|\xi_{2}\right|<W \\ 0 & \text { otherwise }\end{cases}
$$

The corresponding kernel in the direct space is obtained by taking the inverse Fourier transform of this directional filter:

$$
h_{\phi}(\boldsymbol{x})=\int_{\mathbb{R}^{2}} H_{\phi}(\boldsymbol{\xi}) e^{i 2 \pi \boldsymbol{\xi} \cdot \boldsymbol{x}} \mathrm{d} \boldsymbol{\xi} .
$$

For $\phi \in[-\pi / 4, \pi / 4]$, the integral can be split into two parts,

$$
\begin{aligned}
h_{\phi}\left(x_{1}, x_{2}\right)=\int_{-W}^{W}\left[\int_{-W}^{-\xi_{2} \tan \phi}-\left(\xi_{1} \cos \phi+\xi_{2} \sin \phi\right) e^{i 2 \pi\left(\xi_{1} x_{1}+\xi_{2} x_{2}\right)} \mathrm{d} \xi_{1}\right. \\
\left.\quad+\int_{-\xi_{2} \tan \phi}^{W}\left(\xi_{1} \cos \phi+\xi_{2} \sin \phi\right) e^{i 2 \pi\left(\xi_{1} x_{1}+\xi_{2} x_{2}\right)} \mathrm{d} \xi_{1}\right] \mathrm{d} \xi_{2},
\end{aligned}
$$

knowing that $\boldsymbol{\xi} \cdot \boldsymbol{\theta}$ is negative below the line given by $\xi_{1}=-\xi_{2} \tan \phi$ and positive above it. These integrals lead to

$$
\begin{aligned}
& h_{\phi}\left(x_{1}, x_{2}\right)=\left(-x_{2} \cos \phi \operatorname{sinc}\left(\frac{\pi\left(x_{1} \tan \phi-x_{2}\right)}{\tau}\right)-x_{1} \sin \phi \cos \left(\frac{\pi x_{1}}{\tau}\right) \cos \left(\frac{\pi x_{2}}{\tau}\right)\right. \\
& \left.\quad+\left(x_{1} \sin \phi+x_{2} \cos \phi\right) \operatorname{sinc}\left(\frac{\pi x_{2}}{\tau}\right) \cos \left(\frac{\pi x_{1}}{\tau}\right)\right) \frac{1}{2 \tau\left(\pi x_{1}\right)^{2} x_{2}}+\frac{\cos \phi}{2 \tau^{3}} \operatorname{sinc}\left(\frac{\pi x_{1}}{\tau}\right) \operatorname{sinc}\left(\frac{\pi x_{2}}{\tau}\right) .
\end{aligned}
$$

The discretized impulse response, with $x_{1}=m \tau$ and $x_{2}=n \tau$, is given by

$$
h_{\phi}[m, n]= \begin{cases}\frac{1}{2 \pi^{2} \tau^{3}}\left(\frac{-\cos \phi}{m^{2}} \operatorname{sinc}(\pi(m \tan \phi-n))-\cos (\pi n) \cos (\pi m) \frac{\sin \phi}{n m}\right) & \text { if } m, n \neq 0 \\ \frac{\sin ^{2} \phi}{2 \pi^{2} n^{2} \tau^{3} \cos \phi} \cos (\pi n) & \text { if } m=0, n \neq 0 \\ \frac{\cos \phi}{2 \pi^{2} m^{2} \tau^{3}}(\cos (\pi m)-\operatorname{sinc}(\pi m \tan \phi)) & \text { if } m \neq 0, n=0 \\ \frac{2 \cos ^{2} \phi+1}{12 \tau^{3} \cos \phi} & \text { if } m=0, n=0 .\end{cases}
$$



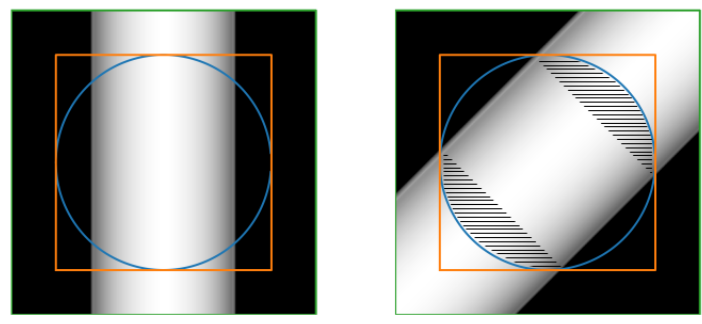

Figure 2: Smeared projection for angle $\phi=0$ (left) and $\phi=-\pi / 4$ (right). The blue circle is the field of view and the orange square is the reconstruction region. The shaded areas represent the regions where the image is truncated along $\boldsymbol{\theta}$. The green square is the region needed to accurately filter the smeared projection for all angles. For $\phi=0$, the data inside the orange square is sufficient for accurate reconstruction inside the FOV, while a larger region is needed for oblique angles, with the largest area being for $\phi=\pi / 4+k \pi / 2$.

The expressions for $m$ and/or $n$ equal to zero are found by setting $x_{1}$ and/or $x_{2}$ to zero in Equation 14. The expression can be extended to angles $\phi \notin[-\pi / 4, \pi / 4]$ using $h_{\phi+\pi}[m, n]=-h_{\phi}[m, n]$ and $h_{\phi+\pi / 2}[m, n]=h_{-\phi}[n, m]$. Note that setting $\phi=0$ in Equation 16 gives the expression of the $1 \mathrm{D}$ ramp filter multiplied by $1 / \tau$ on the line $n=0$, and zero elsewhere. It should also be noted that the impulse response of the filter (Equation 15 has infinite support, such that sampling the filter in the frequency domain would always result in aliasing artifacts according to the sampling theorem (Crawford, 1991).

\subsection{Size of the backprojection region}

In order for the $2 \mathrm{D}$ filtered smeared projections to be equivalent to the $1 \mathrm{D}$ filtered projections, we need non-truncated images $b_{\phi}(\boldsymbol{x})$ along $\boldsymbol{\theta}$ inside the field of view (FOV) (Figure 2). We do not need the full projections outside the FOV since the DR filter tends to a dirac along $\boldsymbol{\theta}^{\perp}$ in the direct space (since it is constant in the Fourier space). By taking a backprojection region (region where the projections will be smeared) of the same size as the reconstruction region, part of the data near the borders will be truncated for angles $\phi \neq k \pi / 2, k \in \mathbb{Z}$. In order to keep the full projections inside a circular FOV for all angles, one should set $M=\sqrt{2} N$, where the backprojection region is $M \times M$ and the reconstruction region is $N \times N$.

\subsection{Simulations}

We generated parallel ray projections of the 2D Shepp Logan phantom (Shepp et al., 1974) using RTK (Rit et al., 2014) in order to test the filter. 804 projections were acquired over a 180 degree range, with a detector of 512 pixels. The phantom was reconstructed using grids of $512 \times 512$ pixels and $1024 \times 1024$ pixels. Poisson noise was applied to the idealized projections, using an initial fluence of $10^{7}$ protons per detector pixel, and weighting the line integrals with $0.01879 \mathrm{~mm}^{-1}$, the linear attenuation coefficient of water at $75 \mathrm{keV}$, to work with physical attenuation values.

A proton CT simulation was performed using the Monte Carlo platform GATE (Jan et al., 2011). The setup is detailed in Rit et al. (2013). A fan beam proton source was simulated, and the list-mode data was binned into parallel geometry images $b_{\phi}(\boldsymbol{x})$ using the same binning as in Rit et al. (2015). The non-linearity of proton trajectories was taken into account by estimating each proton's most likely path (Schulte et al., 2008). We used a spiral phantom, consisting of a disk of water with aluminum inserts (Figure 5). The spatial resolution of each insert was obtained by extracting profiles across the insert edges and determining the frequency corresponding to $10 \%$ of the peak value of the modulation transfer functiion (MTF) of these 

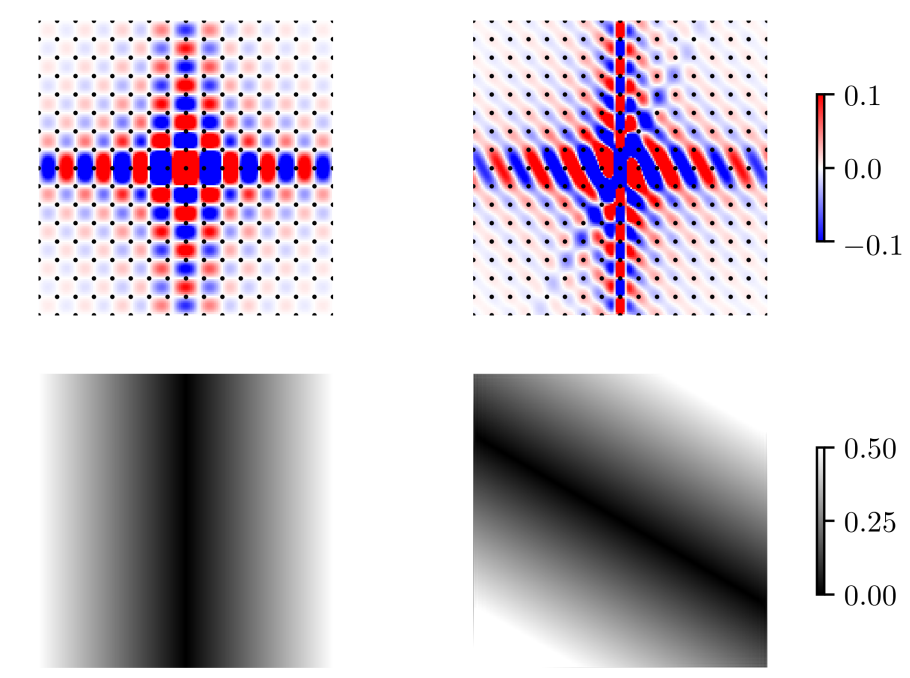

Figure 3: Top : Pseudo-continuous impulse response of the 2D DR filter for $\phi=0$ (left) and $\phi=\pi / 3$ (right). The discrete samples are overlayed as black dots. Bottom : Fourier transform of the discrete impulse response.

profiles. The reconstruction using the DR filter was compared with the algorithm presented in Rit et al. (2013) which generalizes the FBP to non-linear paths.

\section{RESULTS}

Figure 3 shows the "pseudo-continuous" filter, i.e. the oversampled filter calculated from the continuous impulse response (Equation 15) by setting $x_{1}=m \tau^{\prime}$ and $x_{2}=n \tau^{\prime}$ with $\tau^{\prime} \ll \tau$. It is interesting to observe that, after discretization, for the case $\phi=0$ (left), the samples outside the central horizontal line lie exactly on the zero crossings. For the case $\phi=\pi / 3$ (right), the filter is not zero outside the filtering line $n=m \tan \phi$. The DFT of the filters looks like the usual 1D ramp filter duplicated along the direction $\boldsymbol{\theta}^{\perp}$.

The reconstructions of the Shepp-Logan phantom using different parameters are shown in Figure 4. The reconstructed values using the filter sampled in the frequency domain are underestimated. The bias is constant and is equal to -0.17 . The rest of the reconstructions are very similar, although some artifacts are visible near the outer edges of the phantom in the case of the non-apodized DR filter for idealized projections. This is confirmed on the difference maps, where the DR reconstructed image is slightly noisier for idealized projections, although this is only visible with a tight color scale $([-0.01,0.01])$. Using an oversampled backprojection (but keeping the same sampling for the projections) reduces the noise, and apodizing the filter with a Hamming window completely suppresses the artifacts. The reconstructions from noisy projections show the DR filter performs as well as the 1D ramp filter, and that the previous artifacts are hidden by noise.

The reconstructions of proton $\mathrm{CT}$ data are shown in Figure 5. There is no visible difference between the image reconstructed with an FBP and with the DR filter. The spatial resolution $f_{\mathrm{MTF} 10 \%}$ as a function of each bead's distance to the center of the phantom is represented. The difference between the resolution for the FBP and the DR algorithms reaches $0.8 \mathrm{lp} / \mathrm{mm}$ for the outermost bead. 
FBP
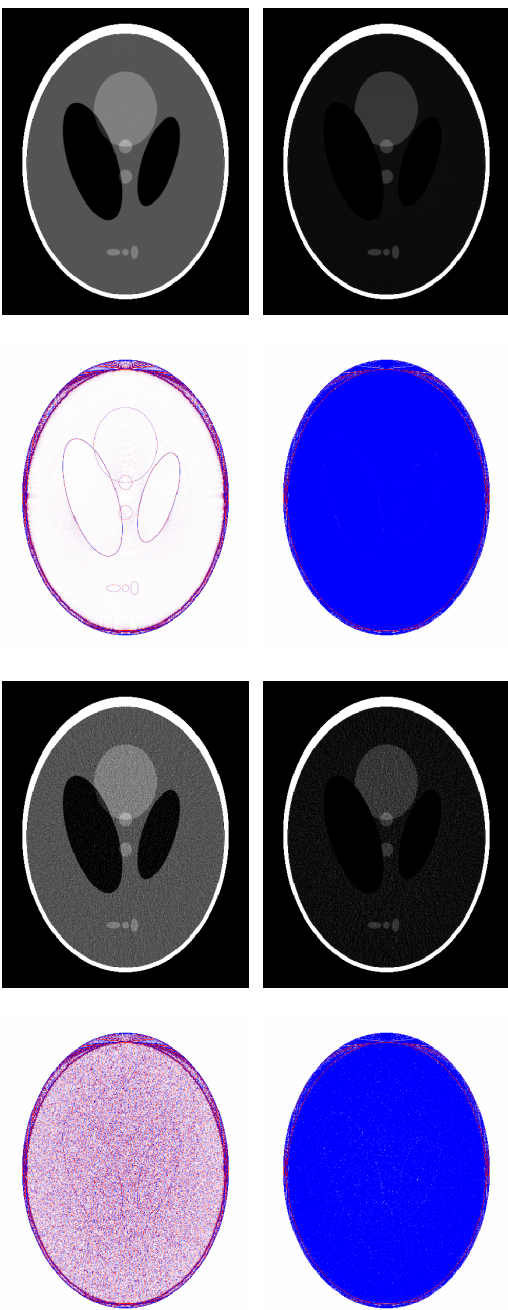

DR (freq samp)
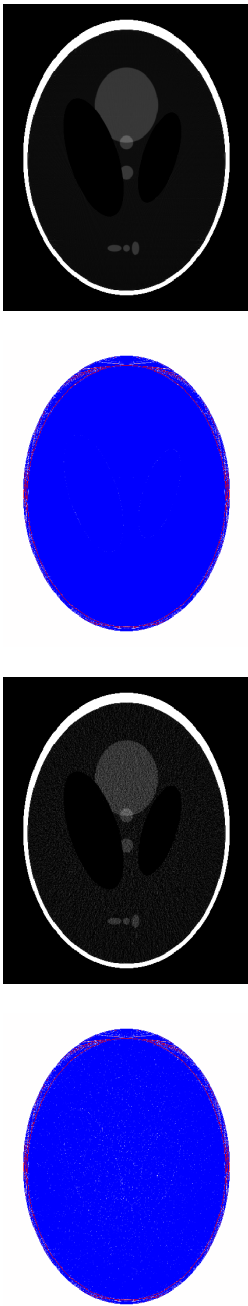
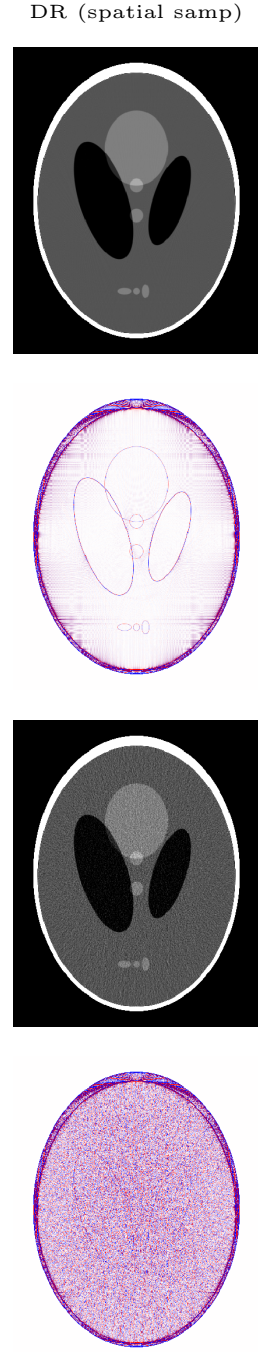

DR oversampled
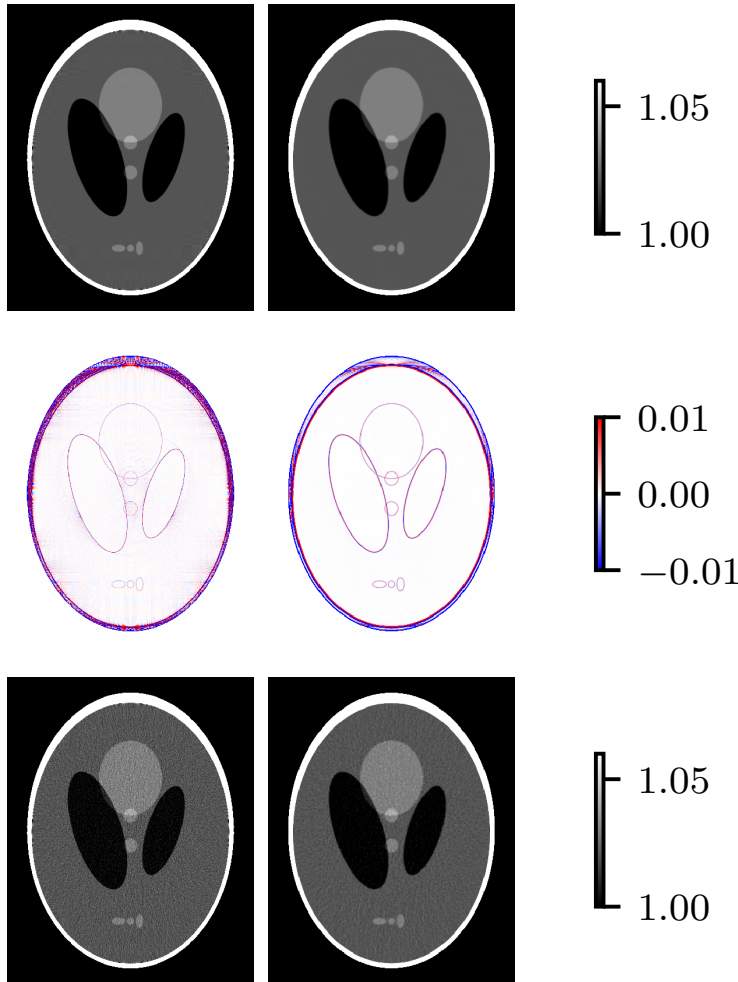
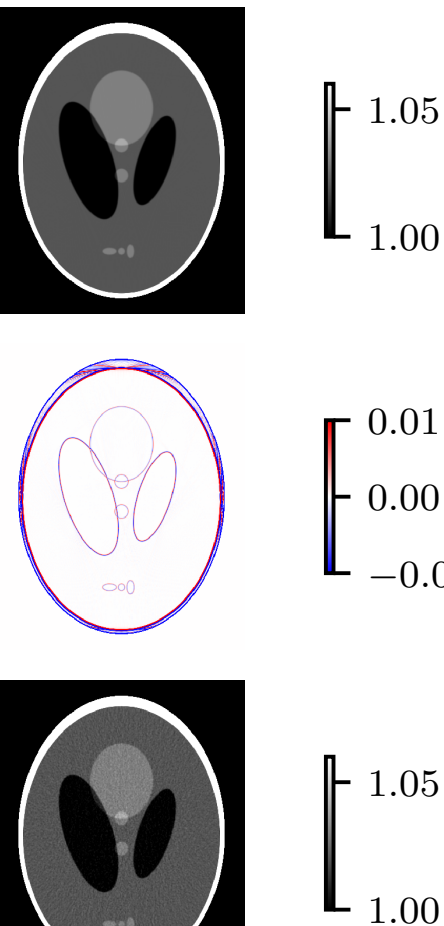

1.00

1.00

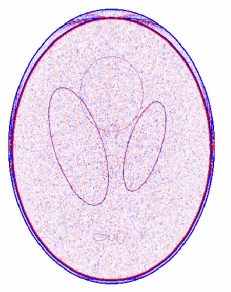

Figure 4: Shepp-Logan phantom reconstructed using, from left to right, an FBP, the DR filter sampled in the frequency and spatial domain, the oversampled DR filter $(\mathrm{N}=1024$, in spatial domain), and the filter apodized with a Hamming window. The first two rows correspond to the results (reconstruction and difference with reference) for idealised projections and the two bottom rows to the results for noisy projections.

\section{DISCUSSION}

Our results show that the 2D DR filter, sampled in the spatial domain, allows accurate reconstruction of $\mathrm{xCT}$ and $\mathrm{pCT}$ data, although some artifacts are apparent in the case of reconstructions from noiseless $\mathrm{xCT}$ projections. We know that, in the direct space, the DR filter should look like a dirac in the direction $\boldsymbol{\theta}^{\perp}$ for the $2 \mathrm{D}$ convolution to be equivalent to the $1 \mathrm{D}$ one. However, apodizing the filter with a square window in Equation 12 results in a convolution with a 2D sinc function, which explains the shape of the filter in Figure 3. While the discretized filter is a dirac for angles $\phi=k \pi / 2, k \in \mathbb{Z}$, since all samples outside the central line are zero, this is not the case for other angles, meaning there will be interferences from other lines during convolution. In Fourier space, this translates in a discontinuity of the FT at the Nyquist frequency. This is visible in Figure 3 where the DFT of the DR filter for $\phi=\pi / 3$ has very thin black lines on its bottom left corner. This could explain the remaining artifacts visible in Figure 4 . A similar issue with the continuity of the FT was reported by Schöndube et al. (2010), where the authors define a 2D inverse Hilbert transform. The image reconstructed using this filter suffered from ring 


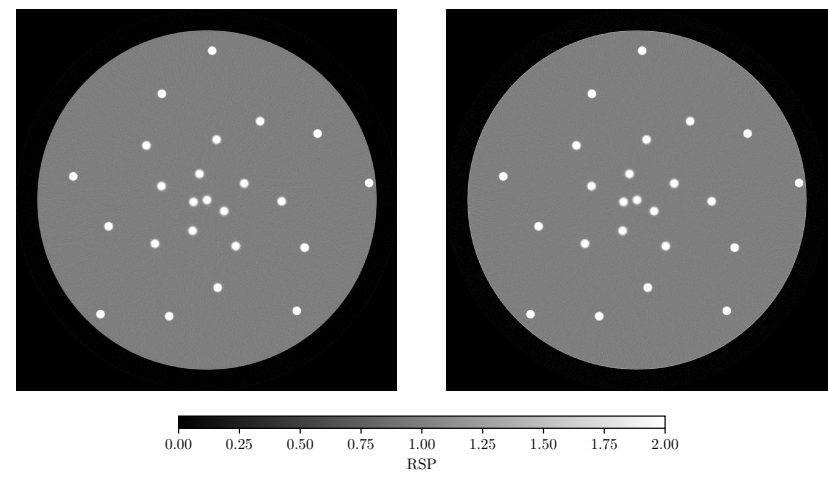

(a)

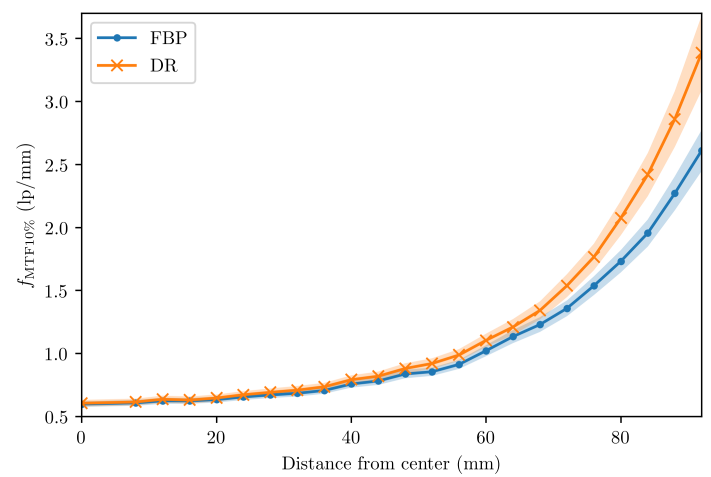

(b)

Figure 5: (a) Proton CT reconstructions of the relative stopping power (RSP) map of the spiral phantom using an FBP (left) and the DR filter (right). (b) Spatial resolution of the inserts in the spiral phantom. The shaded areas correspond to $\pm 3 \sigma$ where $\sigma$ is the uncertainty on the spatial resolution.

artifacts. One of the proposed solutions is to apodize the filter in the Fourier space using a Hamming window to deal with the discontinuity. Our results show that the apodization effectively suppresses artifacts, although it also causes a slight loss of spatial resolution. An alternative is to increase the sampling of the backprojected image (Schöndube et al., 2010), which also reduces the noise as seen in Figure 4. Since the artifacts are related to the sampling of the 2D DR filter, taking more samples i.e. increasing the filter's band limit, will make the reconstruction less noisy. However, this artifact is not visible when using projections with a realistic level of noise. As was observed for the 1D ramp filter by Crawford (1991) and Kak et al. (1988), sampling in the frequency domain will cause aliasing artifacts (an underestimation in the spatial domain) due to the infinite support of the impulse response.

In the case of simple line integrals, such as in $\mathrm{xCT}$, using the 2D DR filter should give the same result as a standard FBP but is computationally less effective. The DR filter could rather be used with modalities such as proton CT or TOF PET, where the projections are respectively integrals along non-linear paths due to multiple Coulomb scattering or integrals along weighted lines due to time resolution of the detector. In $\mathrm{xCT}$, the projections are constant along the direction $\boldsymbol{\theta}^{\perp}$, therefore using 1D projections and the 1D ramp filter makes sense. Both pCT and TOF PET add a new dimension, the depth of the proton or the time-of-flight, such that the smeared projections vary along $\boldsymbol{\theta}^{\perp}$ (see Figure 1). Filtering the 2D smeared projections $b_{\phi}(\boldsymbol{x})$ requires either rotating each image so that its samples are aligned with the $1 \mathrm{D}$ filter, then perform a line-by-line filtering; or using a directional 2D ramp filter. The DR filter could present some notable advantages in this case: as in BPF methods, the filtering is done in the image space so that we avoid rotations that can cause a loss of spatial resolution; at the same time, unlike other $\mathrm{BPF}$ approaches, there is no bias due to the truncation of the backprojection region which does not need to be approximated using very large matrices (2 to 4 times the reconstruction region in Poludniowski et al. (2014) versus $\sqrt{2}$-times). A drawback is that the DR filter is different for each projection angle, but it can be pre-computed for a given image grid and set of projection angles. Our results for proton CT data (Figure 5 have confirmed that the DR filter can improve spatial resolution compared with an FBP algorithm. This improvement was observed only for inserts close to the edge of the spiral phantom because at the center of the object, the image is too blurry due to Coulomb scattering to see any enhancement of spatial resolution.

While this method is theoretically demonstrated for parallel geometries only, the acquisitions can be done in another setup as long as the data is rebinned into parallel geometry, which is straightforward for list-mode $\mathrm{pCT}$ data, as was done for our own $\mathrm{pCT}$ simulations. It may be 
possible to derive an expression for the ramp filter in the case of fan beam data, for example, however, this might prove to be more difficult as the transform from the sinogram domain to the image domain is not a simple rotation as for parallel data.

Another possible use of the DR filter is for 3D parallel projections in $\mathrm{xCT}$, when the detector is rotated by an in-plane angle. The projections would need to be realigned with the image grid before filtering. To avoid this interpolation, the 2D DR filter, computed for the in-plane angle direction, could be used on each 2D projection before backprojection.

\section{CONCLUSION}

We have proposed a 2D version of the ramp filter and applied it to simulated $\mathrm{xCT}$ and $\mathrm{pCT}$ data. This method starts by the smearing of each projection in the image space, then the directiondependent filtering, and finally the angular sum. The filter allows an accurate reconstruction and can be used to avoid an interpolation which would degrade spatial resolution.

\section{Acknowledgements}

The work of Feriel Khellaf was supported by the project DIC20161236452 of the Fondation pour la Recherche Médicale (FRM). The work of Nils Krah was partially funded by the FRM and partially by the European Union's Horizon 2020 research and innovation programme under the Marie Sklodowska-Curie grant agreement No 753370. This work was performed within the framework of the SIRIC LYriCAN INCa_INSERM_DGOS_12563 and the LABEX PRIMES (ANR-11-LABX-0063) of Université de Lyon, within the program "Investissements d'Avenir" (ANR-11-IDEX-0007) operated by the French National Research Agency (ANR). We thank Rolf Clackdoyle, Laurent Desbat and Michel Defrise for useful discussions.

\section{References}

Bracewell, R. N. et al. (1967). "Inversion of fan-beam scans in radio astronomy". In: The Astrophysical Journal 150, p. 427.

Conti, M. et al. (2013). "Estimating image quality for future generations of TOF PET scanners". In: IEEE Transactions on Nuclear Science 60.1, pp. 87-94.

Crawford, C. R. (1991). "CT filtration aliasing artifacts". In: IEEE transactions on medical imaging 10.1, pp. 99-102.

Guo, J. et al. (2011). "Evaluation of windowed image reconstruction for time-of-flight PET". In: 2011 IEEE Nuclear Science Symposium Conference Record. IEEE, pp. 4162-4166.

Jan, S et al. (2011). "GATE V6: a major enhancement of the GATE simulation platform enabling modelling of CT and radiotherapy". In: Physics in Medicine 83 Biology 56.4, p. 881.

Kak, A. C. et al. (1988). Principles of Computerized Tomographic Imaging. New York (USA): IEEE Press.

Kao, C.-M. (2008). "Windowed image reconstruction for time-of-flight positron emission tomography". In: Physics in Medicine 83 Biology 53.13, p. 3431.

Kao, C.-M. et al. (2010). "Analytic reconstruction methods for list-mode time-of-flight PET". In: IEEE Nuclear Science Symposuim \& Medical Imaging Conference. IEEE, pp. 3492-3494.

Noo, F. et al. (2004). "A two-step Hilbert transform method for 2D image reconstruction". In: Physics in Medicine \& Biology 49.17, p. 3903.

Poludniowski, G et al. (2014). "Proton computed tomography reconstruction using a backprojection-then-filtering approach". In: Physics in Medicine $\&$ Biology 59.24, p. 7905.

Ramachandran, G. et al. (1971). "Three-dimensional reconstruction from radiographs and electron micrographs: application of convolutions instead of Fourier transforms". eng. In: Proc Natl Acad Sci USA 68.9, pp. 2236-2240. 
Rit, S. et al. (2013). "Filtered backprojection proton CT reconstruction along most likely paths". In: Medical physics 40.3, p. 031103.

Rit, S. et al. (2014). "The Reconstruction Toolkit (RTK), an open-source cone-beam CT reconstruction toolkit based on the Insight Toolkit (ITK)". In: Journal of Physics: Conference Series. Vol. 489. 1. IOP Publishing, p. 012079.

Rit, S. et al. (2015). "List-mode proton CT reconstruction using their most likely paths via the finite Hilbert transform of the derivative of the backprojection". In: Fully3D, vol. Submitted, pp. 324-327.

Schöndube, H. et al. (2010). "Evaluations of a 2D inverse Hilbert Transform". In: 1st International Conference on Image Formation in X-Ray Computed Tomography. Salt Lake City, USA., p. 80.

Schulte, R. et al. (2008). "A maximum likelihood proton path formalism for application in proton computed tomography". In: Medical physics 35.11, pp. 4849-4856.

Shepp, L. A. et al. (1974). "The Fourier reconstruction of a head section". In: IEEE Transactions on nuclear science 21.3 , pp. 21-43.

Watson, C. C. (2007). "An evaluation of image noise variance for time-of-flight PET". In: IEEE Transactions on Nuclear Science 54.5, pp. 1639-1647.

Zeng, G. L. (2007). "Image reconstruction via the finite Hilbert transform of the derivative of the backprojection". In: Medical physics 34.7, pp. 2837-2843.

Zeng, G. L. et al. (1994). "Can the backprojection filtering algorithm be as accurate as the filtered backprojection algorithm?" In: Proceedings of IEEE Nuclear Science Symposium. Vol. 3. IEEE, pp. 1232-1236. 